\title{
A simple analytical method for heterogeneity corrections in low dose rate prostate brachytherapy
}

\author{
Fernando Hueso-González ${ }^{1,5}$, Javier Vijande ${ }^{1,2}$, \\ Facundo Ballester ${ }^{1}$, Jose Perez-Calatayud ${ }^{3}$ and \\ Frank-André Siebert ${ }^{4}$ \\ ${ }^{1}$ Department of Atomic, Molecular and Nuclear Physics, University of Valencia, Dr. \\ Moliner 50, E-46100 Burjassot, Spain. \\ ${ }^{2}$ IFIC (UV-CSIC), E-46100 Valencia, Spain. \\ ${ }^{3}$ Physics Section, Radiotherapy Department, University and Polytechnic Hospital La \\ Fe, E-46026 Valencia, Spain. \\ ${ }^{4}$ UK S-H, Campus Kiel, Klinik für Strahlentherapie (Radioonkologie), \\ Arnold-Heller-Str. 3, Haus 50, D-24105 Kiel, Germany. \\ ${ }^{5}$ Current address: OncoRay-National Center for Radiation Research in Oncology, \\ Faculty of Medicine and University Hospital Carl Gustav Carus, Technische \\ Universität Dresden, Helmholtz-Zentrum Dresden-Rossendorf, Fetscherstr. 74, PF \\ 41, D-01307 Dresden, Germany.
}

E-mail: Javier.Vijande@uv.es

This is an author-created, un-copyedited version of an article published in Phys. Med. Biol. IOP Publishing Ltd is not responsible for any errors or omissions in this version of the manuscript or any version derived from it. The Version of Record is available online at http://dx.doi.org/10.1088/0031-9155/60/14/5455

1 July 2015

\begin{abstract}
In low energy brachytherapy, the presence of tissue heterogeneities contributes significantly to the discrepancies observed between treatment plan and delivered dose. In this work, we present a simplified analytical dose calculation algorithm for heterogeneous tissue. We compare it with Monte Carlo computations and assess its suitability for integration in clinical treatment planning systems. The algorithm, named as RayStretch, is based on the classic equivalent path length method and TG-43 reference data. Analytical and Monte Carlo dose calculations using Penelope2008 are compared for a benchmark case: a prostate patient with calcifications. The results show a remarkable agreement between simulation and algorithm, the latter having in addition a high calculation speed. The proposed analytical model is compatible with clinical real-time treatment planning systems based on TG-43 consensus datasets for improving dose calculation and treatment quality in heterogeneous tissue. Moreover, the algorithm is applicable for any type of heterogeneities.
\end{abstract}

PACS numbers: 87.53.Jw, 87.55.K, 87.53.Kn, 87.55.-x, 87.55.Gh, 87.53.Bn, 87.19.X-, 87.59.bd

Keywords: brachytherapy, low dose rate, heterogeneities, prostate, calcifications. 


\section{Introduction}

Nowadays, Brachytherapy (BT) is a very important weapon in any oncologist arsenal to manage certain types of cancer. The most usual locations where BT is used are gynaecological, skin, breast, and prostate malignancies. Together with improvements in trans-rectal ultrasound (US) image guidance, permanent low energy (LE) seed implantation has become one of the most successful approaches for treatment of early stage prostate carcinoma (Yu et al. 1999, Pfeiffer et al. 2008).

Current BT treatment planning systems (TPS) allow direct introduction of tabulated dose rates from the literature using the Task Group 43 (TG-43) formalism (Rivard et al. 2007). Consensus datasets for all commercially available photon sources can be found in the joint American Association of Physicists in Medicine (AAPM) and European Society for Radiotherapy and Oncology (ESTRO) reports for high energy (HE) (Perez-Calatayud et al. 2012), and in the AAPM Low Energy Brachytherapy working group supplement to TG-43 (Rivard et al. 2007) for LE. One of the main assumptions behind the TG-43 formalism is the choice of and unbounded water volume as the reference dosimetry medium. This works flawlessly in water-equivalent materials, however it has become evident nowadays that there are certain regions where such approximation might break down.

The reason for such issues can be traced back to fundamental physics considerations simply by focusing on the energy range where the different sources are classified. For LE sources, the photon interaction with matter is mainly dominated by the photoelectric interaction. Such interaction has a strong dependence on the atomic number $(Z)$, and hence on tissue composition. For a HE source, the interaction is governed by Compton scattering; however, the photoelectric interaction might still play a role for high $-Z$ materials, i.e. for shielding considerations. Thus, the implicit all-water approximation for dose calculations using TG-43 formalism is much poorer at low and intermediate energies than at high energies $(E>200 \mathrm{keV})$ (Beaulieu et al. 2012).

Of particular importance is the case of the prostate, where calcifications (accumulation of calcium within a tissue) may appear. Such prostate calculi are rather infrequent below the age of 40 years, however they are common in males over 50 years. They may be solitary but usually occur in clusters located in the posterior and lateral lobes. They are most often asymptomatic, therefore no information regarding its existence is available before imaging the patient. They constitute the main source of heterogeneities found in prostate, both in density and composition. Therefore, LE seed implantation using water-based TG-43 dose distributions might be inaccurate in their vicinity.

Nowadays, the recommended technique (GEC-ESTRO (Salembier et al. 2007), TG137 (Nath et al. 2009), ABS 2012 (Davis et al. 2012)) for prostate implants is an intraoperative procedure using trans-rectal US images. An optimum dose distribution of the implant is obtained by inverse planning techniques using TG-43 data. The outcome of this planning is the number and position of seeds in the prostate. In clinical practice, a 
month later, a dosimetric reassessment of the implant using magnetic resonance (MRI) or computerized tomography (CT) images (the so called post-plan) is performed based on the TPS algorithm. Its aim is to check the positions of the seeds and dosimetry once the oedema has reduced.

In some exceptional cases (not in clinical routine), research groups perform Monte Carlo (MC) simulations based on the post-plan to account for heterogeneities present in the prostate. This effort documents the actual treatment, but there is no possibility to intervene based on its outcome. Hence, the calculations derived from MC in LE brachytherapy do not transfer into a direct benefit to the patient in terms of an optimal plan, however focus exclusively on the accurate knowledge of the dose distribution in an implant already done.

In (Chibani and Williamson 2005), an accelerated MC code was developed for dose calculation in prostate BT. Dosimetric effects of inter-seed attenuation, tissue composition, and tissue calcifications were analysed. The effect caused by the presence of calcifications covering $1 \%-5 \%$ of the prostate volume was discussed. It was found to decrease D80, D90, and D100 values (meaning the minimum dose covering 80\%, 90\% and $100 \%$ of the volume) by up to $32 \%, 37 \%$, and $58 \%$ respectively.

A study was performed in (Landry et al. 2010) to assess the sensitivity of MC dose calculations to uncertainties in human tissue composition for LE BT and electronic sources. They concluded that LE brachytherapy dose distributions in tissue differ from water and are influenced by density, mean tissue composition, and patient-to-patient composition variations. The results support the use of a dose calculation algorithm accounting for heterogeneities such as MC.

The impact of tissue heterogeneities in breast tissue was addressed in (Afsharpour et al. 2010). The impact of tissue heterogeneity and inter-seed attenuation was studied in post-implant evaluation of five clinical permanent breast ${ }^{103} \mathrm{Pd}$ seed implants by means of MC simulations. The authors concluded that the average planning target volume D90 reduction varies from 3.9\% to $35.5 \%$ depending on breast tissue composition. Furthermore, the skin D10 increases by $28.2 \%$ in an entirely adipose breast.

A similar issue was addressed in (Afsharpour et al. 2011). Six modelling schemes including uniform and non-uniform water breast, uniform and non-uniform glandular and adipose mixed tissues, and age-dependent breast, were discussed. The high sensitivity of dosimetry on the modelling scheme reported by the authors argues in favour of an agreement on a standard tissue modelling approach to be used in LE breast brachytherapy.

(Landry et al. 2011) compared $D_{\mathrm{m}, \mathrm{m}}$, where the radiation is transported in the medium and the dose is scored in the same medium, and $D_{\mathrm{w}, \mathrm{m}}$, where the radiation is transported in the medium but the dose is scored in water, obtained from MC simulations for a selection of human tissues of interest using LE low dose rate (LDR) and electronic brachytherapy sources. Large differences were observed between both scoring schemes for a variety of tissues. The authors concluded underlining the need for guidelines on choice of media for dose reporting. 
In (Ghorbani et al. 2014), the effect of tissue composition on dose distribution for different tissues was discussed for ${ }^{103} \mathrm{Pd},{ }^{125} \mathrm{I},{ }^{169} \mathrm{Yb}$, and ${ }^{192} \mathrm{Ir}$ sources. They concluded that TPS considering only a homogeneous treatment volume create errors in dose calculations, the deviation depending on the type of soft tissue, BT source, as well as the distance to the source.

Once the significant impact of heterogeneities and tissue composition is proven, the necessity of complete and detailed dosimetry calculations beyond TG-43 formalism and supported by CT (and MRI) image techniques arises inevitably. The AAPM established a task group (TG-186) to provide guidance for early adopters of modelbased dose calculations algorithms (MBDCAs) for BT to ensure practice uniformity. Such group published a comprehensive report on 2012 (Beaulieu et al. 2012). In some scenarios, dosimetric discrepancies between MBDCAs and water-based TG-43 approaches can reach up to one order of magnitude. Thus, serious concerns were raised and recommendations for a new commissioning procedure of MBDCAs were established.

In summary, in the context of LE BT, the presence of tissue heterogeneities may contribute significantly to dosimetric discrepancies between treatment plan and delivered dose, and several groups are taking efforts to overcome these deviations.

The aim of this paper is to present an analytical algorithm for tissue heterogeneities that could complement the TG-43 formalism used in commercial TPS. As a proof-ofconcept, we study the case of a single seed in a prostate with calcifications (according to an actual patient CT).

\section{Materials and methods}

\subsection{Phantom model}

To analyse the influence of tissue heterogeneities, an anonymized voxelized phantom derived from a prostate patient axial CT scan, see figure 1 (left), provided by La Fe University and Polytechnic Hospital (Valencia, Spain) is selected. La Fe Hospital routinely treats patients using LE BT permanent seed implants, corresponding this patient to a real treatment case. This case is chosen as a benchmark test to illustrate the performance of the algorithm because the patient showed a significant proportion of calcifications inside the prostate. Other cases, not discussed here, have been also analysed.

The CT provides the Hounsfield numbers (HU) in each voxel, hence, the electronic density once a proper calibration curve is given. Unfortunately, this is not enough information to unambiguously determine the composition of a particular voxel. As discussed in Section 1, some assumptions have to be made to identify tissue composition.

Since we are only interested in the calcifications located inside the prostate, we have chosen a minimal scheme: any voxel is either water-equivalent material or a calcification. To do so, we have established an empirical threshold at $100 \mathrm{HU}$. Any voxel with a Hounsfield number under such threshold is considered water, otherwise it 

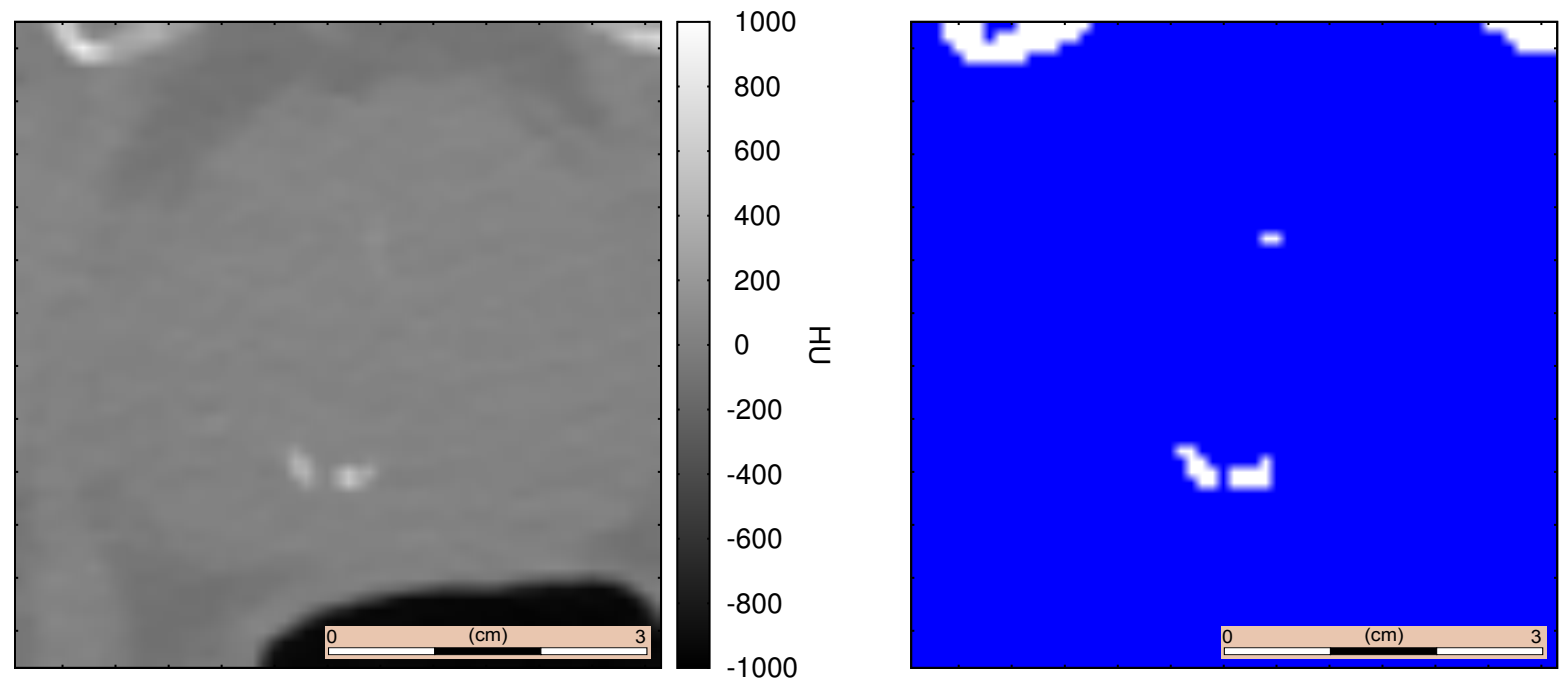

Figure 1. Left: Axial CT scan of a benchmark patient with calcifications inside the prostate (La Fe-Hospital), namely a particular DICOM (Digital Imaging and Communication in Medicine) file corresponding to a transverse image plane $(2.5 \mathrm{~mm}$ slice thickness). Right: Tissue type segmentation (Water-blue, Calcification-white) of the patient $\mathrm{CT}$ according to the calcification threshold, which is set empirically at 100 HU. Three calcifications can be observed at the center of the image.

is a calcification. This can be seen in figure 1; on the left, a CT slice, and on the right, its material decomposition.

For the water composition, we have chosen, as recommended by the TG-43U1, pure degassed liquid water. With respect to the calcification composition, they may vary greatly from patient to patient. As the analysis of composition variability between patients exceeds the scope of this paper, we have selected a typical composition: $\mathrm{H}$ (5.6\%), C (26.5\%), N (3.6\%), O (40.5\%), Na (0.1\%), Mg (0.2\%), P (7.3\%), S (0.3\%) and $\mathrm{Ca}(15.9 \%)$.

The mass density $\rho_{m}$ of each voxel is then calculated by:

$$
\rho_{m}=\left(\frac{\rho_{\mathrm{el}}}{\rho_{\mathrm{el}, \mathrm{w}}}\right) \times \rho_{\mathrm{w}} \times f_{\mathrm{z}, m}
$$

where $\rho_{\mathrm{el}} / \rho_{\mathrm{el}, \mathrm{w}}$ corresponds to the electronic density relative to water provided by the HU calibration curve of La Fe Hospital, $\rho_{\mathrm{w}}=0.998 \mathrm{~g} / \mathrm{cm}^{3}$ is the water mass density in standard conditions and $f_{\mathrm{z}, m}=\overline{(Z / A)}_{\mathrm{w}} / \overline{(Z / A)}_{m}$ is the atomic to mass number quotient in water with respect to the medium $m . f_{\mathrm{z}, \mathrm{w}}=1.0$ for water, whereas for calcifications it will depend on the prescription chosen for evaluating the average $Z$ value. In the present work, we have taken an effective $f_{\mathrm{z}, \mathrm{c}}=0.9$ value for the calcifications. With this prescription, the calcification density will vary depending on the HU between 1.0 and $1.3 \mathrm{~g} / \mathrm{cm}^{3}$ approximately in the region of interest, while the density of water-equivalent prostate tissue lies between 0.85 and $1.1 \mathrm{~g} / \mathrm{cm}^{3}$. Both the tissue type and the mass density will be the voxel-wise input parameters for the MC dose calculations and the analytical algorithm. 


\subsection{Monte Carlo simulation}

A single source model 6711-OncoSeed ${ }^{\mathrm{TM}}$, manufactured by Amersham-Health, is placed at the image center. Such source is widely used for permanent prostate implantation. It consists of a $4.5 \mathrm{~mm}$ welded titanium capsule, $0.05 \mathrm{~mm}$ thick, with welded end caps. The capsule contains a $3.0 \mathrm{~mm}$ long silver rod onto which ${ }^{125} \mathrm{I}$ (mixture of AgBr and AgI in a 2.5:1 molecular ratio) is deposited. Details of the geometry can be found in (Dolan et al. 2006). With regard to this study, large variations for different source models are not expected.

To perform the simulations, we have chosen Penelope2008 (Sempau et al. 2011, Salvat et al. 2008), a MC code whose reliability and performance have been widely tested (Ye et al. 2004). Penelope2008 cross section data are those of the EPDL97 (Cullen et al. 1997) and EEDL (Cullen et al. 2001). For Compton interactions, Penelope2008 uses the impulse approximation to account for Doppler broadening and binding effects in the photon and electron cross-sections. As a consequence, Compton cross-sections in Penelope2008 differ from those of other MC codes. Since the ${ }^{125} \mathrm{I}$ mean energy is $28 \mathrm{keV}$, the possible influence of such a difference is negligible as the dominating process is photoelectric absorption. Recently, a new version of Penelope has been released. Penelope2011 differs with respect to Penelope2008 in the improvements performed in the description of inelastic collisions of electrons and positrons, as well as the representation of nanometric structures. Since these features do not affect the analysis performed in this manuscript, we have used the benchmarked routines developed for Penelope2008 and applied elsewhere (Granero et al. 2011, Vijande et al. 2012, Vijande et al. 2013).

All Monte Carlo simulations in this study conform to the recommendations of sections V.D.2 and V.E of the TG-43U1 report (Rivard et al. 2007) and HEBD report No. 229 (Perez-Calatayud et al. 2012). Following these guidelines, the ${ }^{125}$ I photon spectrum in all MC simulations is taken from the current NuDat database (Kinsey et al. 1996). For the determination of the TG-43 parameters, the source is located at the geometric center of a spherical liquid water phantom with $40 \mathrm{~cm}$ radius to estimate dose to water and simulate unbounded phantom conditions for $r<20 \mathrm{~cm}$. Its composition and mass density are those recommended by AAPM (mass density of $0.998 \mathrm{~g} / \mathrm{cm}^{3}$ at $22^{\circ} \mathrm{C}$ ) (Rivard et al. 2007). In order to provide adequate spatial resolution, cells are: $\Delta r=0.1 \mathrm{~mm}$ voxels for distances $r \leq 1 \mathrm{~cm}, \Delta r=0.5 \mathrm{~mm}$ voxels for $1 \mathrm{~cm}<r \leq 5 \mathrm{~cm}$, $\Delta r=1 \mathrm{~mm}$ voxels for $5 \mathrm{~cm}<r \leq 10 \mathrm{~cm}$, and $\Delta r=2 \mathrm{~mm}$ voxels for $10 \mathrm{~cm}<r \leq 20 \mathrm{~cm}$, where $r$ is defined as the distance from the center of the active part of the source. Angular sampling resolution is $1^{\circ}$. Additional simulations are performed as recommended by AAPM to obtain the air kerma strength $S_{\mathrm{K}}$ with the source surrounded by vacuum except for a cylindrical air cell of size $0.1 \mathrm{~cm} \times 0.1 \mathrm{~cm}$ at $r=10 \mathrm{~cm}$.

The DICOM file containing the phantom model described in Subsection 2.1 has been translated into Penelope2008 format using a home-made routine. Once imported, collisional kerma using linear track-length estimator (Williamson 1987) and absorbed dose are scored. For the case of collisional kerma, home-made routines are specifically 
developed. Both estimators are evaluated in a voxel grid taken from the DICOM information: $\Delta x=\Delta y=0.977 \mathrm{~mm}$ and $\Delta z=2.5 \mathrm{~mm}$, where $\Delta x \times \Delta y$ corresponds to the CT pixel size and $\Delta z$ to the CT slice thickness. Therefore, voxels have a constant volume of $2.386 \mathrm{~mm}^{3}$. The number of photons generated in each simulation is $5 \times 10^{9}$, obtaining dose uncertainties (coverage factor $k=2$ ) about $0.01 \%$ on the prostate volume. Due to the energy of the ${ }^{125}$ I photon spectrum, electronic equilibrium is reached even in regions in the vicinity of the sources, hence, the dose can be approximated by collisional kerma even for small distances from the source capsule. Therefore, in the following, only collisional kerma will be reported.

\subsection{Analytical algorithm RayStretch}

We define an effective dose function that depends on the parameters of the heterogeneity and the reference values in water, as an alternative to a computationally intensive MC simulation on a patient basis. Thanks to our analytical algorithm, named as RayStretch, which is based on the classic water-equivalent path length method and TG-43 reference data, a real time dose recalculation accounting for heterogeneities in a clinical case is expected to be feasible.

Conservation of energy We start from the law of conservation of energy: a seed imparts on average an energy $\epsilon$ within a volume $V$ during its complete radioactive lifetime. If the volume is large enough, there will be no energy leakage and the identity holds:

$$
\epsilon_{V}(\mathrm{w})=\epsilon_{V}(\text { het })
$$

where ' $\mathrm{w}$ ' refers to water, that is, the homogeneous case, whereas 'het' refers to the heterogeneous case, e.g. a calcification inside a water volume.

From the definition of absorbed dose $D$ at any position $\vec{r}$ in a mass $\mathrm{d} M$ (volume $\mathrm{d} V$ ) during the full source lifetime:

$$
D(\vec{r})=\frac{\mathrm{d} \epsilon}{\mathrm{d} M} \Longrightarrow \mathrm{d} \epsilon=D(\vec{r}) \mathrm{d} M=D(\vec{r}) \rho(\vec{r}) \mathrm{d} V
$$

where $\rho(\vec{r})$ is the mass density. As the energy is conserved, see equation $(2)$, it follows:

$$
\int_{V} D_{\mathrm{w}}(\vec{r}) \rho_{\mathrm{w}} \mathrm{d} V=\int_{V} D_{\text {het }}(\vec{r}) \rho_{\text {het }}(\vec{r}) \mathrm{d} V
$$

Note that the seed is located at the origin of the coordinate system $\vec{r}_{s}=\overrightarrow{0}$. If we assume a tissue with spherical symmetry, the problem depends only on the radial coordinate $r=|\vec{r}|$ (the distance to the seed). We rename the integral of the angular part as $\int_{0}^{4 \pi} D(\vec{r}) d \Omega \equiv 4 \pi D(r)$ and obtain:

$$
\int_{0}^{\infty} D_{\mathrm{w}}(r) \rho_{\mathrm{w}} r^{2} \mathrm{~d} r=\int_{0}^{\infty} D_{\text {het }}(r) \rho_{\text {het }}(r) r^{2} \mathrm{~d} r
$$


The dose rate $\dot{D}_{\mathrm{w}}$ (dose absorbed in unit time) in the TG-43 formalism, considering the one-dimensional, point source approximation and random orientation (Baltas et al. 2006), is defined as:

$$
\dot{D}_{\mathrm{w}}(r)=S_{\mathrm{K}} \Lambda g_{\mathrm{p}, \mathrm{w}}(r) \varphi_{\mathrm{an}}(r)\left(\frac{r_{0}}{r}\right)^{2}
$$

where $\varphi_{\text {an }}(r)$ is the anisotropy factor, $\Lambda$ the dose rate constant, $S_{\mathrm{K}}$ the air kerma strength, $g_{\mathrm{p}, \mathrm{w}}(r)$ the radial dose function (dimensionless), and $r_{0}$ a reference distance of $1 \mathrm{~cm}$. We define $\dot{D}_{\text {het }}$ analogously to the dose rate in water:

$$
\dot{D}_{\text {het }}(r)=S_{\mathrm{K}} \Lambda g_{\mathrm{p}, \text { het }}(r) \varphi_{\mathrm{an}}(r)\left(\frac{r_{0}}{r}\right)^{2}
$$

where we assume that the anisotropy factor in water $\varphi_{\mathrm{an}, \mathrm{w}}(r) \approx \varphi_{\mathrm{an}, \mathrm{het}}(r) \equiv \varphi_{\mathrm{an}}(r)$ is similar to the heterogeneous case.

We consider an exponential decay of the source's activity with mean life $\tau$, so that the time dependence can be integrated independently.

$$
D(r)=\int_{0}^{\infty} \dot{D}(r) e^{-t / \tau} \mathrm{d} t=\dot{D}(r) \tau
$$

After substituting equations (6), (7) and (8) in identity (5) and cancelling constant factors $\left(S_{\mathrm{K}}, \Lambda, r_{0}, \tau\right)$ appearing on both sides, we obtain:

$$
\rho_{\mathrm{w}} \int_{0}^{\infty} g_{\mathrm{p}, \mathrm{w}}(r) \varphi_{\mathrm{an}}(r) \mathrm{d} r=\int_{0}^{\infty} \rho_{\text {het }}(r) g_{\mathrm{p}, \text { het }}(r) \varphi_{\mathrm{an}}(r) \mathrm{d} r
$$

This purely formal derivation is the background of our algorithm, that has to estimate the real dose $D_{\text {het }}$ in the heterogeneous case by means of an effective radial dose function $g_{\mathrm{p}, \text { het }}(r)$.

Water equivalent path Let us assume a geometric differential segment in water $\Delta r$. A photon crossing a segment of the same length in another medium will not deposit the same energy as in water. For this reason, the classic water equivalent length $\Delta r_{\text {eq }}$ is defined as a virtual segment in water, where the photon would release the same amount of energy as in the real segment $\Delta r$ in the given medium. In general $\Delta r_{\text {eq }}=\lambda \Delta r$, where $\lambda$ is a scaling factor of the radial differential segment.

This scaling factor has to be adjusted empirically by comparing the algorithm with the MC simulation. Nevertheless, one could relate it physically with the mass density and the interaction cross section and parametrize it appropriately. Assuming a radial exponential attenuation of the uncollided photons, the mean free path is given by $1 / \mu$ (where $\mu$ is the linear attenuation coefficient). Consequently, the radial scaling factor $\lambda$ can be interpreted as the quotient between mean free paths in the corresponding media at the mean energy value $\bar{E}$ of the photon:

$$
\lambda=\frac{1 / \mu_{\mathrm{w}, \bar{E}}}{1 / \mu_{\mathrm{het}, \bar{E}}}=\frac{\mu_{\mathrm{het}, \bar{E}}}{\mu_{\mathrm{w}, \bar{E}}}
$$


One can introduce the Mass Attenuation Coefficients (Hubbell and Seltzer 2004), where $\rho$ is the mass density. It follows:

$$
\lambda=\frac{(\mu / \rho)_{\mathrm{het}, \bar{E}}}{(\mu / \rho)_{\mathrm{w}, \bar{E}}} \frac{\rho_{\mathrm{het}}}{\rho_{\mathrm{w}}}
$$

Hence, the equivalent path is proportional to the relative mass density $\rho_{\text {het }} / \rho_{\mathrm{w}}$ and to the relative mass attenuation coefficients.

Energy redistribution Eqs. (2) and (9) describe the conservation of energy in the whole volume. Nonetheless, we need to retrieve the energy deposit at each point. Thanks to the equivalent path method, we can locally account for a greater energy deposition in tissues with higher mass density and interaction cross section than water.

To put it another way, we transform a medium with higher density into a medium with normal density but scaled (larger) distances, and associate the energy released along a path in the heterogeneity to the energy released in a longer path in water (where the dose is given by the reference values).

This is illustrated with a simple spherical tissue model, similar to an onion with three layers:

- From $r=0$ to $r=a$, it is water.

- From $r=a$ to $r=b$, there is a calcification with a thickness $t=b-a$.

- For $r>b$, it is water.

The heterogeneity has a constant mass density $\rho_{\mathrm{c}}>\rho_{\mathrm{w}}$ and higher mass attenuation coefficient, so that $\lambda>1$. In this scenario, we divide the region in differential steps and assume that the energy $E$ released between $r$ and $r+\Delta r$ equals the energy released in water between the equivalent path limits $r_{\mathrm{eq}}$ and $(r+\Delta r)_{\mathrm{eq}}$, as depicted in figure 2. Note that $r_{\text {eq }}(r \leq a)=r, r_{\text {eq }}(a<r<b)=a+\lambda(r-a)$ and $r_{\text {eq }}(r \geq b)=a+\lambda(b-a)+(r-b)$. $\Delta r_{\text {eq }}=\lambda \Delta r$ for $a<r<b, \Delta r_{\text {eq }}=\Delta r$ otherwise. Compared to equation (3), the law of conservation of energy is applied here differentially:

$$
\left.E_{\mathrm{het}}\right|_{r} ^{r+\Delta r}=\left.E_{\mathrm{w}}\right|_{r_{\mathrm{eq}}} ^{r_{\mathrm{eq}}+\Delta r_{\mathrm{eq}}}
$$

$E_{\mathrm{w}}$ is the energy released in water and $E_{\mathrm{het}}$ in the heterogeneous medium. The latter is related with the integral of the radial dose function through equation (9), but in this case restricting the infinite volume to differential integration limits:

$$
\int_{r}^{r+\Delta r} \rho\left(r^{\prime}\right) g_{\mathrm{p}, \mathrm{eq}}\left(r^{\prime}\right) \varphi_{\mathrm{an}}\left(r^{\prime}\right) \mathrm{d} r^{\prime}=\rho_{\mathrm{w}} \int_{r_{\mathrm{eq}}}^{r_{\mathrm{eq}}+\Delta r_{\mathrm{eq}}} g_{\mathrm{p}, \mathrm{w}}\left(r^{\prime}\right) \varphi_{\mathrm{an}}\left(r^{\prime}\right) \mathrm{d} r^{\prime}
$$

where $\rho\left(r^{\prime}\right)$ is either $\rho_{\mathrm{w}}$ or $\rho_{\mathrm{c}}$. We also simplify the notation by changing 'het' with 'eq' (it stands for equivalent) and $g_{\mathrm{p}, x}$ with $g_{x}$. For small enough $\Delta r$, the left side 
of the identity can be integrated directly and, after reorganizing terms, we obtain the equivalent radial dose function:

$$
g_{\mathrm{eq}}(r)=\frac{\rho_{\mathrm{w}}}{\rho(r)} \frac{1}{\Delta r} \int_{r_{\mathrm{eq}}}^{r_{\mathrm{eq}}+\Delta r_{\mathrm{eq}}} g_{\mathrm{w}}\left(r^{\prime}\right) \frac{\varphi_{\mathrm{an}}\left(r^{\prime}\right)}{\varphi_{\mathrm{an}}(r)} \mathrm{d} r^{\prime}
$$

For the sake of clarity, and motivated by the small absolute variation of the anisotropy factor with distance (Dolan et al. 2006), we assume $\varphi_{\text {an }}\left(r^{\prime}\right) \approx \varphi_{\text {an }}\left(r_{\text {eq }}\right)$ as constant inside the integration limits and approximate the quotient $\varphi_{\text {an }}\left(r_{\text {eq }}\right) / \varphi_{\text {an }}(r) \approx 1$. Hence, the simplified version of equation 14 yields:

$$
g_{\mathrm{eq}}(r)=\frac{\rho_{\mathrm{w}}}{\rho(r)} \frac{1}{\Delta r} \int_{r_{\mathrm{eq}}}^{r_{\mathrm{eq}}+\Delta r_{\mathrm{eq}}} g_{\mathrm{w}}\left(r^{\prime}\right) \mathrm{d} r^{\prime}
$$

Finally, the effective dose is given by equation (7). Therefore, with this approach, the dose is obtained from TG-43 consensus data stored on a TPS according to the radial dose function for water $g_{\mathrm{w}}$ integrated around the equivalent distance $r_{\text {eq }}$, whereas the anisotropy factor is not altered.

Discretization We need to adapt our equations to the voxelized phantom instead of just a spherical three-layer tissue model. The distance $r$ between calculation point (center of voxel $j$ ) and source location is connected by a virtual straight line which crosses a finite set of voxels $\{i\}$. This distance $r$ has to be scaled according to the relative mass density and mass attenuation coefficient of the voxels crossed by the connecting line. For this reason, ray tracing inside the phantom is required (Siddon 1985).

Let us order spatially and numerate all intersecting voxels $\{i\}$, and denote the corresponding segment (length of intersection) inside each voxel as $\Delta r(i)$. For each line segment, the scaling factor of equation (11) is applied to obtain the water equivalent voxel step $\Delta r_{\text {eq }}(i)$ :

$$
\Delta r_{\mathrm{eq}}(i)=\Delta r(i) \frac{\rho_{i}}{\rho_{\mathrm{w}}} \frac{(\mu / \rho)_{i}}{(\mu / \rho)_{\mathrm{w}}}
$$

$\rho_{\mathrm{w}}$ and $(\mu / \rho)_{\mathrm{w}}$ are the mass density and mass attenuation coefficient in water, whereas the subscript $i$ refers to those quantities in voxel $i$. We omit the subindex $\bar{E}$ for the sake of clarity.

When adding over all intersecting voxels $\{i\}$, we obtain the global equivalent distance:

$$
r_{\mathrm{eq}}(r ;\{i\})=\sum_{i} \Delta r_{\mathrm{eq}}(i)=\sum_{i} \Delta r(i) \frac{\rho_{i}}{\rho_{\mathrm{w}}} \frac{(\mu / \rho)_{i}}{(\mu / \rho)_{\mathrm{w}}}
$$

Note that for the last voxel of the ordered set (voxel $j$, the calculation point), the intersection $\Delta r_{j}$ has to be divided by two in the summation, as the segment length $r$ goes only until the voxel center (the middle point of the intersection of voxel with virtual prolonged line). Idem for the first voxel intersection $\Delta r_{0}$ depending on the seed center position. 


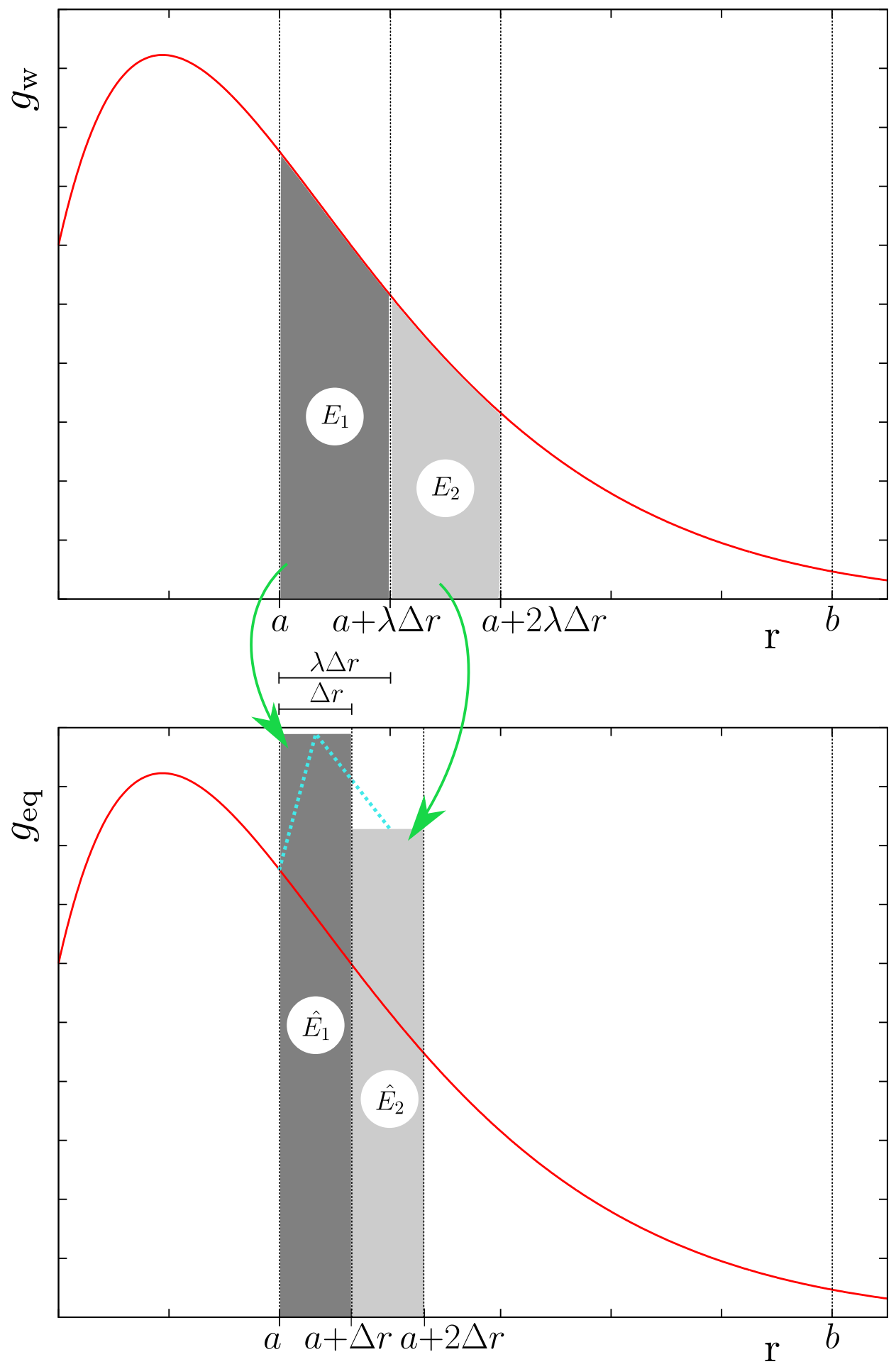

Figure 2. Schematic illustration of the redistribution of energy inside the heterogeneity. The blue dotted line represents the construction of the equivalent dose function $g_{\text {eq }}$ by transforming the reference curve $g$ (water) with the radial scaling factor $\lambda$, see equation (11) of the algorithm. The area $E_{i}$ is not directly the energy, see equation $(9)$, thus $E_{i} \neq \hat{E}_{i}$. 
For the energy redistribution, see equation (12), we chose these lengths of intersection $\Delta r(i)$ as differential steps. Thus, the energy $E_{j}$ deposited in a certain voxel $j$ whose central point is separated a distance $r$ from the radioactive seed (crossing a set of voxels $\{i\})$ and with a last intersecting segment $\Delta r(j)$ is:

$$
\left.E_{j}\right|_{r-\Delta r(j) / 2} ^{r+\Delta r(j) / 2}=\left.E_{\mathrm{w}}\right|_{r_{\mathrm{eq}}-\Delta r_{\mathrm{eq}}(j) / 2} ^{r_{\mathrm{eq}}+\Delta r_{\mathrm{eq}}(j) / 2}
$$

After developing equation (18) in analogy to (15), the equivalent radial dose function $g_{\text {eq }}(r)$ for voxel $j$ yields:

$$
g_{\mathrm{eq}}(r)=\frac{\rho_{\mathrm{w}}}{\rho_{j}} \frac{1}{\Delta r(j)} \int_{r_{\mathrm{eq}}-\Delta r_{\mathrm{eq}}(j) / 2}^{r_{\mathrm{eq}}+\Delta r_{\mathrm{eq}}(j) / 2} g_{\mathrm{w}}\left(r^{\prime}\right) \mathrm{d} r^{\prime}
$$

In summary, the calculated dose depends on the following input parameters of the algorithm for the whole voxel set $\{i\}$ :

- The voxel mass density $\rho_{i}$ (from the CT and calibration curves).

- The voxel mass attenuation coefficient $(\mu / \rho)_{i}$ at mean energy value $\bar{E}$ (from the CT-guided tissue segmentation and empirical calibration).

- The radial dose function in water $g_{\mathrm{w}}(r)$ and anisotropy factor $\varphi_{\mathrm{an}}(r)$ (obtained from TG-43 consensus data and implemented into the TPS).

- The dose rate constant $\Lambda$ (obtained from TG-43 consensus data and implemented into the TPS).

- The air kerma strength $S_{\mathrm{K}}$ (TPS).

\section{Results}

The role played by calcifications in the dosimetric characterization of a realistic case is depicted in figure 3 for one particular transverse image plane in the DICOM volume. Dose rate times its distance to the source squared $\dot{D} \times\left|\vec{r}-\overrightarrow{r_{s}}\right|^{2} \equiv \dot{D} r^{2}$ divided by the air kerma strength is shown in cGy $\mathrm{mm}^{2} \mathrm{~h}^{-1} \mathrm{U}^{-1}$. Figure 3(a) shows the results of a detailed MC simulation, whereas 3(b) depicts those obtained using RayStretch, the algorithm proposed in Section 2.3 .

The quotient between heat maps b) and a) is shown in figure 4 and compared with the quotient between RayStretch algorithm and the TG-43 based calculation.

Three profiles are shown in figure 5 to illustrate some interesting cases. In the image on the left of each profile, the line actually drawn across the two-dimensional (2D) map is shown in white.

\section{Discussion}

In figure 3, the severe impact due to the presence of calcifications in the dosimetry as compared with the homogeneous case can be seen. TG-43 based calculation fails to 
$\mathrm{MC}$ simulation (a)

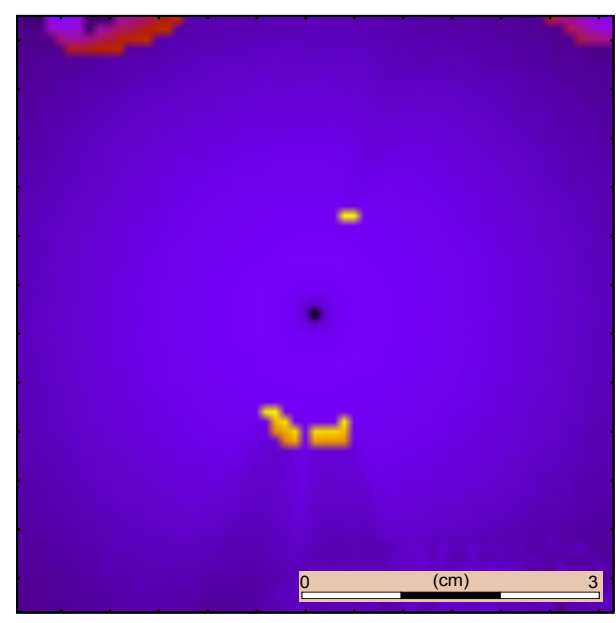

Algorithm (b)

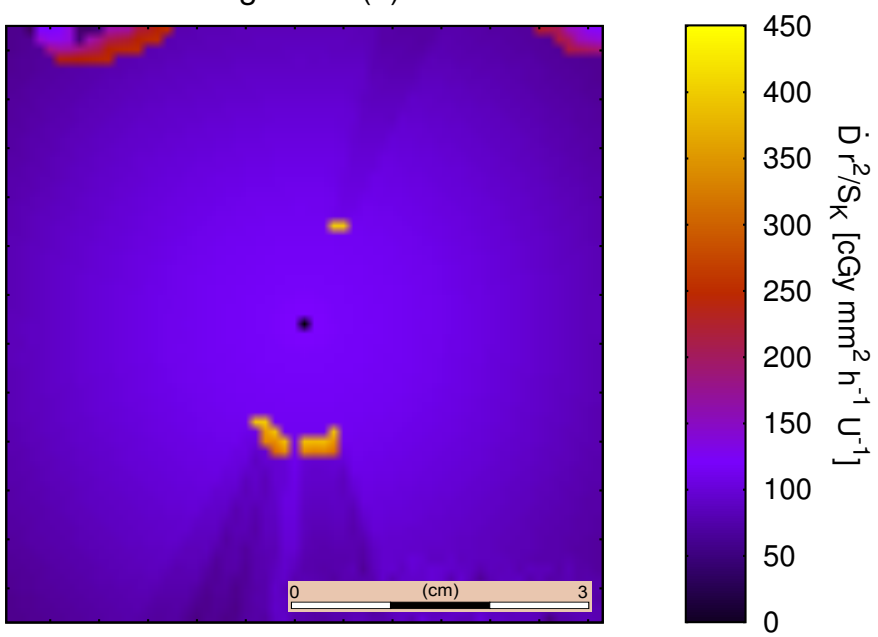

Figure 3. Dose rate by air kerma strength multiplied by the squared distance to the radioactive seed (located at the image center) for (a) MC simulation, and (b) analytical algorithm RayStretch. Heat maps are shown in a common color scale range for a reliable comparison.
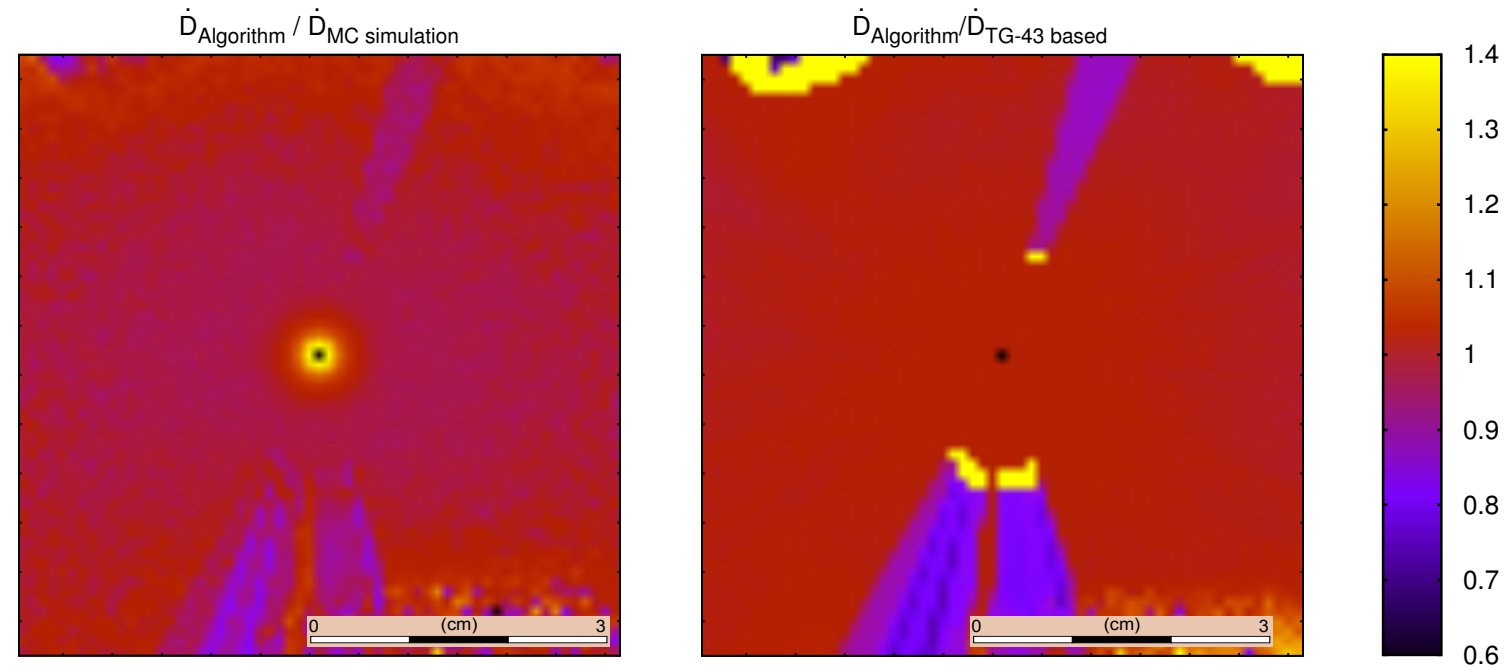

Figure 4. Quotient of the dose rate calculated by the RayStretch algorithm and the MC simulation (left), and the algorithm divided by the TG-43 based calculation (right). Color scale is common for both maps and is adapted to the range of interest (values outside the palette range have the same color as the closest boundary color).

incorporate any patient-related details into the dose rate map, however, both the MC simulation and the proposed algorithm reproduce details of the anatomy by using the information contained in the DICOM file.

In both approaches, hot spots, regions where the absorbed dose is higher than the one predicted by TG-43, can be observed in calcificated regions and bones due to the higher absorption coefficient of these materials. Cold spots in the form of shadows can be seen behind calcificated regions and bones as a consequence of the attenuation suffered when crossing the denser regions. 


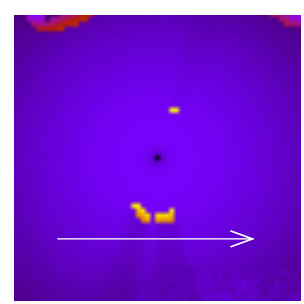

A

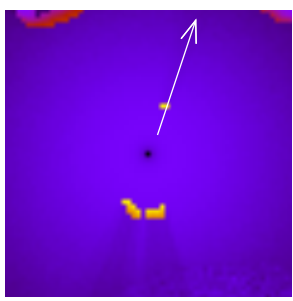

B
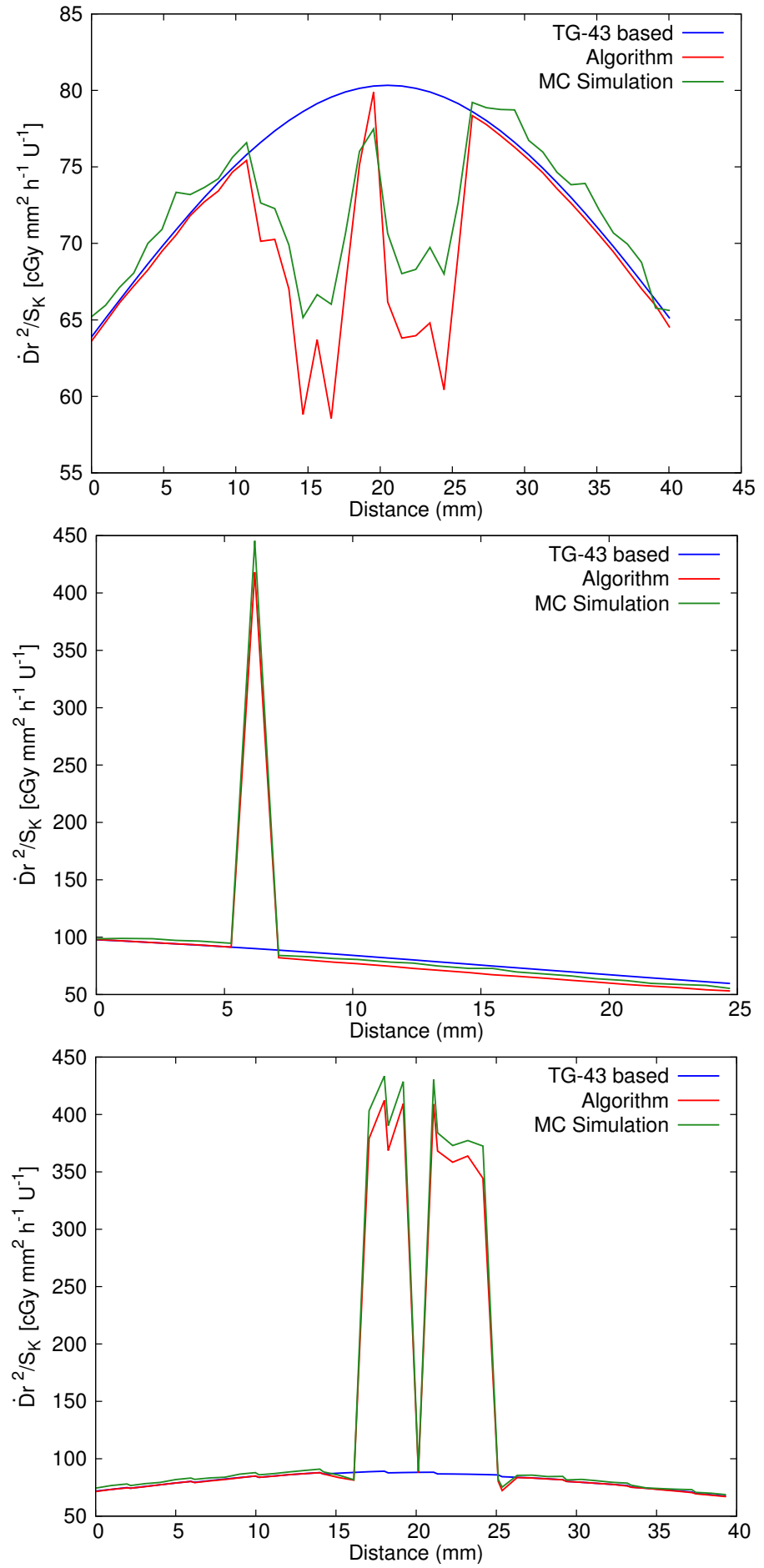

Figure 5. $\dot{D} \times\left|\vec{r}-\overrightarrow{r_{s}}\right|^{2} \equiv \dot{D} \times r^{2}$ profiles (divided by $S_{\mathrm{K}}$ ), where $\overrightarrow{r_{s}}$ stands for the source position. The TG-43 based calculation (blue) is compared to the RayStretch algorithm (red) and the MC simulation (green). The insert in the top left corner of each profile shows the line (white arrow) drawn across the 2D dose map of figure 3 . The horizontal axis refers to the local distance within the white arrow according to its starting point and direction. 
In figure 1 -left, one can identify an air-filled part of the rectum. The dose shown in figure 3 for this volume is not correct since neither the simulation nor the analytical algorithm include the description of air-filled volumes at this stage of the study.

Some discrepancies are observed between algorithm and simulation in figure 4-left. In the voxels around the radioactive seed, high deviations are found as the algorithm does not account for the source thickness. Leaving aside this local effect, the discrepancies remain below $20 \%$ in all the regions of interest and are only evident in the cold spots. These differences are very sensitive to the average mass absorption coefficient of equation (11), which is empirically adjusted. Nevertheless, assuming MC as the gold standard, the algorithm provides a much more faithful dosimetric description than the TG-43 based calculation and reduces significantly the discrepancies (figure 4 -right) behind the calcification.

This effect is clearly seen in the line profiles depicted in figure 5 for three regions of interest which illustrate the effect on the dose produced by tissue heterogeneities:

- A: Dose cold spot caused by two prostate calcifications between seed and calculation point.

- B and C: Dose hot spot effects in prostate calcifications.

Based on figure 5, it can be concluded that the agreement between RayStretch and simulation is remarkable for most of the regions evaluated. A more detailed conversion of CT to tissue composition and mass density as well as the introduction of an energy dependent instead of average mass absorption coefficient may have the potential to reduce the remaining deviations.

Concerning computation performance, the dose is calculated with a desktop computer for a single seed and a grid of $61 \times 61 \times 24=89304$ voxels, corresponding to a cube of $6 \times 6 \times 6 \mathrm{~cm}^{3}$; the computation time is $0.14 \mathrm{~s}$ for the analytical algorithm (on a single central processing unit) and $100 \mathrm{~h}$ for the MC simulation with $5 \times 10^{9}$ events.

\section{Conclusions}

RayStretch, a simplified analytical algorithm for dose calculation in heterogeneous tissue, is described and compared with Penelope2008 simulations for a benchmark case: an actual prostate patient with calcifications. Simulated dose calculations are in remarkable agreement with the analytical algorithm, which is based on the water equivalent path length and on TG-43 consensus datasets. In addition, the algorithm is applicable for any type of heterogeneities and has the potential for real-time computation. Due to the current limitations of the TG-43 based calculations for low dose rate brachytherapy during the implant, even if the agreement with simulations is not complete, any improvement in the real-time dosimetric evaluation is welcome.

In conclusion, thanks to its compatibility with clinical TPS and its high computation speed, RayStretch is a promising candidate for improving real-time dose calculation and treatment quality in heterogeneous tissue during prostate implants. 


\section{Acknowledgments}

This study was partly supported by a fellowship grant from the Spanish Ministry of Education, by the Generalitat Valenciana under Project PROMETEOII/2013/010, by the Spanish Government under Project No. FIS2013-42156 and by the European Commission within the Seventh Framework Program through ENTERVISION (grant agreement number 264552).

\section{References}

Afsharpour, H., Landry, G., Reniers, B., Pignol, J.-P., Beaulieu, L. and Verhaegen, F. (2011). Tissue modeling schemes in low energy breast brachytherapy, Phys. Med. Biol. 56(22): 7045.

URL: http://dx.doi.org/10.1088/0031-9155/56/22/004

Afsharpour, H., Pignol, J.-P., Keller, B., Carrier, J.-F., Reniers, B., Verhaegen, F. and Beaulieu, L. (2010). Influence of breast composition and interseed attenuation in dose calculations for post-implant assessment of permanent breast ${ }^{103} \mathrm{Pd}$ seed implant, Phys. Med. Biol. 55(16): 4547.

URL: http://dx.doi.org/10.1088/0031-9155/55/16/S09

Baltas, D., Sakelliou, L. and Zamboglou, N. (2006). The Physics of Modern Brachytherapy for Oncology, Series in Medical Physics and Biomedical Engineering, Taylor \& Francis.

URL: $h t t p: / / d x . d o i . o r g / 10.1201 / 9781420012422$

Beaulieu, L., Carlsson Tedgren, A., Carrier, J.-F., Davis, S. D., Mourtada, F., Rivard, M. J., Thomson, R. M., Verhaegen, F., Wareing, T. A. and Williamson, J. F. (2012). Report of the Task Group 186 on model-based dose calculation methods in brachytherapy beyond the TG-43 formalism: current status and recommendations for clinical implementation, Med. Phys. 39(10): 6208-6236.

URL: $h t t p: / / d x . d o i . o r g / 10.1118 / 1.4747264$

Chibani, O. and Williamson, F. (2005). MCPI@): A sub-minute Monte Carlo dose calculation engine for prostate implants, Med. Phys. 32(12): 3688-3698.

URL: $h t t p: / / d x . d o i . o r g / 10.1118 / 1.2126822$

Cullen, D., Hubbell, J. and Kissel, L. (1997). EPDL97: the Evaluated Photon Data Library, '97 Version, Lawrence Livermore National Laboratory. Report No.: UCRL-50400 6(Rev. 5).

URL: http://citeseerx.ist.psu.edu/viewdoc/summary?doi=10.1.1.196.8287

Cullen, D., Perkins, S. and Seltzer, S. (2001). Tables and graphs of electron-interaction crosssections from $10 \mathrm{eV}$ to $100 \mathrm{GeV}$ derived from the LLNL Evaluated Electron Data Library (EEDL) Z=1100, Lawrence Livermore National Laboratory. Contract No.: UCRL-50400 . URL: $h t t p: / / d x . d o i . o r g / 10.2172 / 5691165$

Davis, B. J., Horwitz, E. M., Lee, W. R., Crook, J. M., Stock, R. G., Merrick, G. S., Butler, W. M., Grimm, P. D., Stone, N. N., Potters, L., Zietman, A. L. and Zelefsky, M. J. (2012). American Brachytherapy Society consensus guidelines for transrectal ultrasound-guided permanent prostate brachytherapy, Brachytherapy 11(1): 6 - 19.

URL: $h t t p: / / d x . d o i . o r g / 10.1016 / j . b r a c h y .2011 .07 .005$

Dolan, J., Li, Z. and Williamson, J. F. (2006). Monte Carlo and experimental dosimetry of an I-125 brachytherapy seed, Med. Phys. 33(12): 4675-4684.

URL: $h t t p: / / d x . d o i . o r g / 10.1118 / 1.2388158$

Ghorbani, M., Salahshour, F., Haghparast, A., Moghaddas, T. A. and Knaup, C. (2014). Effect of tissue composition on dose distribution in brachytherapy with various photon emitting sources, Journal of Contemporary Brachytherapy 6(1): 54-67.

URL: $h t t p: / / d x . d o i . o r g / 10.5114 / j c b .2014 .42024$

Granero, D., Vijande, J., Ballester, F. and Rivard, M. J. (2011). Dosimetry revisited for the HDR 
${ }^{192}$ Ir brachytherapy source model mHDR-v2, Med. Phys. 38(1): 487-494.

URL: $h t t p: / / d x . d o i . o r g / 10.1118 / 1.3531973$

Hubbell, J. H. and Seltzer, S. M. (2004). Tables of X-Ray Mass Attenuation Coefficients and Mass Energy-Absorption Coefficients from $1 \mathrm{keV}$ to $20 \mathrm{MeV}$ for Elements $\mathrm{Z}=1$ to 92 and 48 Additional Substances of Dosimetric Interest, NIST .

URL: http://www.nist.gov/pml/data/xraycoef/

Kinsey, R. R., Dunford, C. L., Tuli, J. K. and Burrows, T. W. (1996). The NUDAT/PCNUDAT Program for Nuclear Data, 9th International Symposium of Capture Gamma-Ray Spectroscopy and Related Topics, Vol. Data extracted from the NUDAT database, version May 2014.

URL: http://www.nndc.bnl.gov/

Landry, G., Reniers, B., Murrer, L., Lutgens, L., Bloemen-Van Gurp, E., Pignol, J.-P., Keller, B., Beaulieu, L. and Verhaegen, F. (2010). Sensitivity of low energy brachytherapy Monte Carlo dose calculations to uncertainties in human tissue composition, Med. Phys. 37(10): 51885198.

URL: $h t t p: / / d x$. doi.org/10.1118/1.3477161

Landry, G., Reniers, B., Pignol, J.-P., Beaulieu, L. and Verhaegen, F. (2011). The difference of scoring dose to water or tissues in Monte Carlo dose calculations for low energy brachytherapy photon sources, Med. Phys. 38(3): 1526-1533.

URL: $h t t p: / / d x . d o i . o r g / 10.1118 / 1.3549760$

Nath, R., Bice, W. S., Butler, W. M., Chen, Z., Meigooni, A. S., Narayana, V., Rivard, M. J. and $\mathrm{Yu}, \mathrm{Y}$. (2009). AAPM recommendations on dose prescription and reporting methods for permanent interstitial brachytherapy for prostate cancer: Report of Task Group 137, Med. Phys. 36(11): 5310-5322.

URL: $h t t p: / / d x$. doi.org/10.1118/1.3246613

Perez-Calatayud, J., Ballester, F., Das, R. K., DeWerd, L. A., Ibbott, G. S., Meigooni, A. S., Ouhib, Z., Rivard, M. J., Sloboda, R. S. and Williamson, J. F. (2012). Dose calculation for photon-emitting brachytherapy sources with average energy higher than $50 \mathrm{keV}$ : Report of the AAPM and ESTRO, Med. Phys. 39(5): 2904-2929.

URL: $h$ ttp://dx.doi.org/10.1118/1.3703892

Pfeiffer, D., Sutlief, S., Feng, W., Pierce, H. M. and Kofler, J. (2008). AAPM Task Group 128: Quality assurance tests for prostate brachytherapy ultrasound systems, Med. Phys. 35(12): 5471-5489.

URL: $h t t p: / / d x . d o i . o r g / 10.1118 / 1.3006337$

Rivard, M. J., Butler, W. M., DeWerd, L. A., Huq, M. S., Ibbott, G. S., Meigooni, A. S., Melhus, C. S., Mitch, M. G., Nath, R. and Williamson, J. F. (2007). Supplement to the 2004 update of the AAPM task group no. 43 report, Med. Phys. 34(6): 2187-2205.

URL: $h t t p: / / d x . d o i . o r g / 10.1118 / 1.2736790$

Salembier, C., Lavagnini, P., Nickers, P., Mangili, P., Rijnders, A., Polo, A., Venselaar, J. and Hoskin, P. (2007). Tumour and target volumes in permanent prostate brachytherapy: A supplement to the ESTRO/EAU/EORTC recommendations on prostate brachytherapy, Radiotherapy and Oncology 83(1): 3-10.

URL: http://dx.doi.org/10.1016/j.radonc.2007.01.014

Salvat, F., Fernandez-Varea, J. and Sempau, J. (2008). PENELOPE-2008, a code system for Monte Carlo simulation of electron and photon transport, Issy-les-Moulineaux, France .

URL: https://www.oecd-nea.org/science/pubs/2009/nea6416-penelope.pdf

Sempau, J., Badal, A. and Brualla, L. (2011). A PENELOPE-based system for the automated Monte Carlo simulation of clinacs and voxelized geometries-application to far-from-axis fields, Med. Phys. 38(11): 5887-5895.

URL: $h$ ttp://dx.doi.org/10.1118/1.3643029

Siddon, R. L. (1985). Fast calculation of the exact radiological path for a threedimensional CT 
array, Med. Phys. 12(2): 252-255.

URL: http://dx.doi.org/10.1118/1.595715

Vijande, J., Granero, D., Perez-Calatayud, J. and Ballester, F. (2012). Monte Carlo dosimetric study of the Flexisource Co-60 high dose rate source, Journal of Contemporary Brachytherapy 4(1): 34-44.

URL: $h t t p: / / d x . d o i . o r g / 10.5114 / j c b .2012 .27950$

Vijande, J., Granero, D., Perez-Calatayud, J. and Ballester, F. (2013). Monte Carlo dosimetric study of the medium dose rate CSM40 source, Applied Radiation and Isotopes 82(0): 283288.

URL: http://dx.doi.org/10.1016/j.apradiso.2013.09.003

Williamson, J. (1987). Monte-Carlo evaluation of kerma at a point for photon transport problems, Med. Phys. 14(4): 567-576.

URL: $h t t p: / / d x . d o i . o r g / 10.1118 / 1.596069$

Ye, S., Brezovich, I., Pareek, P. and Naqvi, S. (2004). Benchmark of PENELOPE code for lowenergy photon transport: dose comparisons with MCNP4 and EGS4, Phys. Med. Biol. 49(3): 387-397.

URL: $h t t p: / / d x . d o i . o r g / 10.1088 / 0031-9155 / 49 / 3 / 003$

Yu, Y., Anderson, L., Li, Z., Mellenberg, D., Nath, R., Schell, M., Waterman, F., Wu, A. and Blasko, J. (1999). Permanent prostate seed implant brachytherapy: Report of the American Association of Physicists in Medicine Task Group No. 64, Med. Phys. 26(10): 2054-2076.

URL: http://dx.doi.org/10.1118/1.598721 